\title{
KIF18B promotes breast cancer cell proliferation, migration and invasion by targeting TRIP13 and activating the Wnt/ $\beta$-catenin signaling pathway
}

\author{
LAN LIU $^{1}$, ZHAOFENG ZHANG ${ }^{1}$, XIULIN XIA ${ }^{1}$ and JING LEI ${ }^{2}$ \\ Departments of ${ }^{1}$ Mammary Glands and ${ }^{2}$ Women's Healthcare, Baoji Maternal and \\ Child Health Hospital, Baoji, Shaanxi 721000, P.R. China
}

Received August 20, 2021; Accepted December 8, 2021

DOI: $10.3892 / \mathrm{ol} .2022 .13232$

\begin{abstract}
Kinesin superfamily member 18B (KIF18B) has previously been reported to be upregulated in breast cancer (BC) and is involved in BC tumorigenesis. Therefore, the present study aimed to investigate the effects and underlying mechanisms of KIF18B in BC. Comprehensive bioinformatics analysis was performed, using data from The Cancer Genome Atlas. KIF18B knockdown and thyroid hormone receptor-interacting protein 13 (TRIP13) overexpression in $\mathrm{BC}$ cells were induced via transfection, by using the short hairpin RNA-KIF18B and overexpression-TRIP13 vectors, respectively. Cellular processes, including proliferation, migration and invasion were assessed using colony formation, wound healing and Transwell assays, respectively. mRNA and protein expression levels were determined using reverse transcription-quantitative PCR and western blot analysis, respectively. Protein-protein interactions were determined using co-immunoprecipitation. The results demonstrated that the KIF18B expression levels were upregulated in BC, particularly in triple-negative BC (TNBC) tissues and cell lines. KIF18B knockdown inhibited the proliferation, migration and invasion of HCC-1937 TNBC cells. Furthermore, MMP12 and MMP9 protein expression levels were decreased by KIF18B knockdown. TRIP13 expression was also demonstrated to be upregulated in BC, particularly in TNBC tissues and cell lines. TRIP13 expression levels positively correlated with those of $\mathrm{KIF} 18 \mathrm{~B}$ in BC tissues and cells, and further analysis verified that TRIP13 and KIF14B were able to directly bind to each other. However, TRIP13 overexpression abolished the effects of KIF18B knockdown on HCC-1937 cells. Furthermore, KIF18B knockdown decreased $\beta$-catenin, c-Myc and cyclin
\end{abstract}

Correspondence to: Dr Jing Lei, Department of Women's Healthcare, Baoji Maternal and Child Health Hospital, 2 Xinjian Road, Baoji, Shaanxi 721000, P.R. China

E-mail: leijing812@163.com

Key words: breast cancer, kinesin superfamily member 18B, thyroid hormone receptor-interacting protein $13, \mathrm{Wnt} / \beta$-catenin
D1 protein expression levels; however, TRIP13 overexpression resulted in the recovery of all respective protein expression levels. On the whole, the present study demonstrates that KIF18B promotes BC malignant events, including the proliferation, migration and invasion of TNBC cells. These results indicate that KIF18B may play an oncogenic role in $\mathrm{BC}$ by upregulating TRIP13 expression, thereby activating the Wnt/ $\beta$-catenin signaling pathway.

\section{Introduction}

Breast cancer (BC) occurs due to the uncontrolled proliferation of breast epithelial cells, ultimately becoming malignant, as a result of various carcinogens (1). BC treatment strategies include surgery, radiotherapy and systemic therapy, which are usually based on a multimodal approach, depending on the stage and biology of the tumor, as well as patient acceptance and tolerance (2). Advances in the early detection of novel effective biomarkers, technologies of oncoplastic surgery, and improvement in patients' lifestyle have contributed to improvements in the outcomes of patients with $\mathrm{BC}(3,4)$; however, patients who develop metastatic $\mathrm{BC}$ and drug resistance continue to have a poor prognosis (5). Therefore, novel approaches for $\mathrm{BC}$ therapeutics, including the reduction of $\mathrm{BC}$ relapse and the improvement of the mortality rate are urgently required.

The kinesin superfamily (KIFs) is a group of proteins characterized by their instrumental role in the intracellular transport of microtubule-based chromosomes during mitosis (6). The dysregulation of KIFs may result in uncontrolled cell proliferation, resulting from premature sister chromatid separation, highlighting their importance in tumorigenesis. In total, 45 KIFs with various functions have been discovered and identified in humans, among which numerous family members have been reported to play a crucial role in tumor pathobiology (7). Furthermore, a previous study demonstrated the involvement of KIFs in BC metastasis, prognosis and resistance to chemotherapy (8). KIF member 18B (KIF18B) is a KIF superfamily member. Recent studies have suggested that KIF18B expression may be significantly upregulated in BC tissue and is closely associated with a worse overall, relapse-free and distant metastasis-free survival in BC $(9,10)$. It has also been demonstrated in another study that KIF18B silencing 
may restrict cell proliferation and invasion, and enhance $\mathrm{BC}$ chemosensitivity by modulating the Akt/GSK-3 $\beta / \beta$-catenin signaling pathway (11). Thus, it was hypothesized that KIF18B may exert a tumor-promoting effect on $\mathrm{BC}$. However, the potential regulatory mechanisms of KIF18B in BC have not yet been fully elucidated.

The thyroid hormone receptor-interacting protein 13 (TRIP13) is an ATPase protein associated with various cellular activities, playing a crucial role in cell progression, particularly in checkpoint signaling (12). Over the past few decades, the oncogenic role of TRIP13 has attracted considerable attention. It has been reported that TRIP13 may be upregulated in numerous types of cancer and is usually associated with a poor prognosis (13). Moreover, TRIP13 expression is upregulated in $\mathrm{BC}$ and is significantly associated with numerous clinicopathological features, also indicating a poor prognosis of patients with BC $(14,15)$.

In the present study, KIF18B expression was predicted to positively correlate with TRIP13 expression in BC via analysis using The Cancer Genome Atlas (TCGA) database. The aim of the present study was therefore to investigate whether KIF18B promotes the malignant progression of $\mathrm{BC}$ by regulating TRIP13.

\section{Materials and methods}

Cell culture. The human normal breast epithelial MCF-10A and MCF-12A cell lines, which have been widely used as control cell lines for BC cell lines (16,17), and BC MCF-7, ZR-75-1, HCC-1937 and MDA-MB-436 cell lines, were purchased from the American Type Culture Collection (ATCC). Cells were cultured at $37^{\circ} \mathrm{C}$ in DMEM (Thermo Fisher Scientific, Inc.) supplemented with 10\% FBS (Gibco; Thermo Fisher Scientific, Inc.), in a humid atmosphere with $5 \% \mathrm{CO}_{2}$.

Cell transfection. The KIF18B-targeting short hairpin RNA (shRNA) oligonucleotide sequences and TRIP13 cDNA sequences were designed and synthesized by GenScript and were cloned into the pSilencer 2.1-U6 Neo and pcDNA3.1 vectors (Thermo Fisher Scientific, Inc.), respectively, which were consequently named shRNA-KIF18B-1 (5'-GAGACC TCAATGCCACCTTTG-3'), shRNA-KIF18B-2 (5'-CCAGTT TCCATGAATGCATTG-3') and overexpressed (Ov)-TRIP13. shRNA-negative control (NC; 5'-ATGGCGATTCGTATC ATGGCAT-3') and empty pcDNA3.1 vector were used as NCs. Transfection was performed using Lipofectamine $2000^{\circledR}$ (Thermo Fisher Scientific, Inc.) according to the manufacturer's protocol at $37^{\circ} \mathrm{C}$ for $24 \mathrm{~h}$. At $48 \mathrm{~h}$ following transfection, the transfection efficiency was measured using reverse transcription-quantitative PCR (RT-qPCR) and western blot analysis.

$R T-q P C R$. Total RNA was extracted from the HCC-1937 cells using the Total RNA Extraction kit (Beijing Solarbio Science \& Technology Co., Ltd.; R1200) according to the manufacturer's protocol. Total RNA was reverse transcribed using the PrimeScript RT Reagent kit (Takara Biotechnology Co., Ltd.; cat. no. RR047A) according to the manufacturer's protocol. qPCR was performed using the TB Green PrimeScript RT-PCR kit (Takara Biotechnology Co., Ltd.; RR086A) and a StepOnePlus Real-Time PCR system (Applied Biosystems; Thermo Fisher Scientific, Inc.) according to the manufacturer's protocol. The following thermocycling conditions were used for the qPCR: initial denaturation at $95^{\circ} \mathrm{C}$ for $30 \mathrm{sec}$; and 40 cycles of denaturation at $95^{\circ} \mathrm{C}$ for $10 \mathrm{sec}$, annealing at $60^{\circ} \mathrm{C}$ for $20 \mathrm{sec}$ and extension at $70^{\circ} \mathrm{C}$ for $10 \mathrm{sec}$. The primer pair sequences are listed in Table I. mRNA expression levels were quantified using the $2^{-\Delta \Delta \mathrm{Cq}}$ method (18) and normalized to the internal reference gene GAPDH.

Western blot analysis. Transfected or untransfected HCC-1937 cells were lysed using RIPA lysis buffer (Vazyme Biotech Co., Ltd.) according to the manufacturer's protocol. The total protein concentration was determined using a BCA Protein Quantification kit (Beyotime Institute of Biotechnology). Total protein (50 $\mu \mathrm{g}$ protein/lane) from each sample was separated via SDS-PAGE on a $12 \%$ gel and transferred onto PVDF membranes. Following blocking with 5\% non-fat milk at room temperature for $2 \mathrm{~h}$, all membranes were subsequently incubated with primary antibodies at $4^{\circ} \mathrm{C}$ overnight. Following primary antibody incubation, the membranes were incubated with HRP-conjugated anti-rabbit secondary antibody (1:10,000; ab6721, Abcam) for a further $2 \mathrm{~h}$ at room temperature. Protein bands were visualized using ChemiGlow detection reagents (ProteinSimple). The blots were then visualized using a FluorChem 8900 Imager and semi-quantified using ImageJ software (V1.8.0; National Institutes of Health). The following primary antibodies were purchased which were obtained from Abcam: KIF18B (1:5,000; ab168812), TRIP13 (1:5,000; ab128153), MMP12 (1:10,000; ab52897), MMP9 $(1: 10,000 ; a b 76003), \beta$-catenin $(1: 5,000 ; a b 32572)$, c-Myc $(1: 1,000 ; a b 32072)$, cyclin D1 (1:200; ab16663) and GAPDH (1:10,000; ab181602). GADPH was used as the loading control.

Cell proliferation assay. The Cell Counting Kit-8 (CCK-8) assay (Beyotime Institute of Biotechnology) was used to detect cell proliferation. Briefly, transfected or untransfected cells were seeded into a 96 -well plate $\left(1 \times 10^{3}\right.$ cells/well) and cultured at $37^{\circ} \mathrm{C}$ in $5 \% \mathrm{CO}_{2}$. Following incubation for 24,48 and $72 \mathrm{~h}$, $10 \mu \mathrm{l}$ CCK-8 reagent were added to the culture solution and incubated for a further $1 \mathrm{~h}$. Cell proliferation was assessed using a microplate reader (Bio-Rad Laboratories, Inc.), based on the absorbance at $450 \mathrm{~nm}$.

Colony formation assay. In order to observe cell proliferation, the HCC-1937 cells were seeded into a $6-\mathrm{cm}$ plate at a density of 500 cells/plate and cultured for $24 \mathrm{~h}$. Subsequently, the culture medium was replaced with fresh DMEM and cultured for a further 14 days. Colonies were infiltrated with methanol for $10 \mathrm{~min}$, stained with crystal violet for $10 \mathrm{~min}$ at room temperature, imaged using a fluorescence microscope (magnification, x100; Olympus Corporation) and counted using ImageJ software (V1.8.0; National Institutes of Health).

Wound healing assay. To evaluate cell migration, transfected and untransfected HCC-1937 cells were seeded into 6-well plated at a density of $1 \times 10^{4}$ cells/well. When cells had reached almost $100 \%$ confluency, wounds were created in the cell monolayers using a sterile pipette tip. Subsequently, the cells were incubated in serum-free medium for $24 \mathrm{~h}$ at $37^{\circ} \mathrm{C}$. The 
Table I. Primer sequences for RT-quantitative PCR.

\begin{tabular}{lll} 
Gene name & \multicolumn{1}{c}{ Forward (5'-3') } & \multicolumn{1}{c}{ Reverse $\left(5^{\prime}-3^{\prime}\right)$} \\
\hline$K I F 18 B$ & GGAGAACCGACGAAAGGTGT & AGGGTTAAACACCAGCACC-C \\
TRIP13 & TGCTGATTGATGAGGTGGAGAG & GGTTGCACAAGTATCACGCA \\
GAPDH & ATGGGCAGCCGTTAG-GAAAG & CCCAATACGACCAAATCAGAGAA
\end{tabular}

wounds were imaged at 0 and $24 \mathrm{~h}$, using an inverted microscope (magnification, x200; Olympus Corporation). ImageJ software (V1.8.0; National Institutes of Health) was used to determine wound widths.

Transwell invasion assay. A 24-well Transwell System (Costar; Corning, Inc.) was employed to evaluate the invasive ability of the HCC-1937 cells following transfection. Cells were seeded into the upper chambers which were pre-coated with $5 \mu \mathrm{l}$ Matrigel $^{\mathrm{TM}}$ (BD Biosciences) at $37^{\circ} \mathrm{C}$ for $30 \mathrm{~min}$ at a density of $1 \times 10^{5}$ cells/chamber with serum-free medium. The lower chamber was filled with $500 \mu 1$ DMEM containing $10 \%$ FBS. Following incubation for $24 \mathrm{~h}$ at $37^{\circ} \mathrm{C}$, invading cells in the lower chamber were fixed using formalin for $30 \mathrm{~min}$ and stained with $0.5 \%$ crystal violet (MilliporeSigma) for $15 \mathrm{~min}$ at room temperature. Cells were imaged using a light microscope and counted using a high-powered microscope (magnification, x200).

Co-immunoprecipitation (Co-IP). The HCC-1937 cells were harvested and lysed using RIPA lysis buffer (Beyotime Institute of Biotechnology). Protein lysates were incubated with anti-KIF18B (1:1,000; ab168812, Abcam) and anti-TRIP13 antibodies $(1: 1,000 ;$ ab128153, Abcam) or normal rabbit IgG $\left(1: 1,000 ;\right.$ ab172730, Abcam) and rotated overnight at $4^{\circ} \mathrm{C}$ for the formation of an immune-complex. The reaction mixture was then incubated with protein A/G plus-agarose beads (Santa Cruz Biotechnology, Inc.) for $2 \mathrm{~h}$ at $4^{\circ} \mathrm{C}$. After being washed five times with cold washing buffer, the agarose beads were heated with $40 \mu 110 \%$ SDS buffer to $100^{\circ} \mathrm{C}$ for $5 \mathrm{~min}$. The samples were analyzed using western blot analysis, as described above.

Statistical analysis. Data were represented by at least three independent experimental repeats and are presented as the mean \pm SD. Statistical analysis was performed using GraphPad Prism 7.0 (GraphPad Software, Inc.). Comparisons between two groups were analyzed using the unpaired Student's t-test. One-way ANOVA followed by Tukey's post hoc test was used to calculate the statistical differences between $\geq 3$ groups. Pearson's Correlation analysis was used to analyze the correlation between two genes expression in $\mathrm{BC}$. $\mathrm{P}<0.05$ was considered to indicate a statistically significant difference.

\section{Results}

KIF18B expression is upregulated in BC. KIF18B expression profile in BC was analyzed by using TCGA data and the UALCAN database. The results demonstrated that KIF18B expression was upregulated in patients with $\mathrm{BC}$, particularly in patients with the triple-negative BC (TNBC) subtype, in comparison with healthy individuals (Fig. 1A and B).
Moreover, the KIF18B expression levels in human normal breast cells (MCF-10A and MCF-12A), and in the BC cell lines, MCF-7, ZR-75-1, HCC-1937 and MDA-MB-436, were also evaluated. The results demonstrated that the $\mathrm{BC}$ cell lines had significantly higher KIF18B mRNA and protein expression levels in comparison with the normal breast epithelial cells (Fig. 1C and D). The TNBC HCC-1937 cell line displayed the highest KIF18B expression levels and was thus selected for use in subsequent experiments.

Knockdown of KIF18B inhibits the malignant progression of $B C$ cells. KIF18B expression was knocked down in HCC-1937 BC cells using shRNAs. shRNA-KIF18B-1 was selected for use in following experiments as a result of its greater transfection efficiency (Fig. 2A and B). Following KIF1B knockdown, the results demonstrated the decreased viability of the HCC-1937 cells (Fig. 2C). The colony formation assay demonstrated that the KIF1B knockdown group exhibited a markedly reduced number of colonies compared with the NC group (Fig. 2D). Furthermore, HCC-1937 cell migratory and invasive abilities were markedly suppressed as a result of KIF18B knockdown (Fig. 2E and F). Moreover, KIF18B knockdown decreased the MMP12 and MMP9 protein expression levels (Fig. 2G), which have both been reported to be involved in tumor cell invasion and metastasis (19).

TRIP13 expression is upregulated in BC and is positively regulated by the direct binding of KIF18B. Following TCGA data analysis using the UALCAN database, the results demonstrated that KIF18 expression positively correlated with TRIP13 expression in $\mathrm{BC}$, with a Pearson correlation coefficient of 0.65 (Fig. 3A). Furthermore, TRIP13 expression was found to be upregulated in patients with $\mathrm{BC}$, particularly in patients with TNBC (Fig. 3B and C). To further verify this finding, TRIP13 expression in normal breast epithelial MCF-10A and MCF-12A cells and BC MCF-7, ZR-75-1, HCC-1937 and MDA-MB-436 cells was investigated. Results demonstrated that TRIP13 exhibited higher expression levels in BC cell lines, especially in the HCC-1937 cell line, indicating the potential role of TRIP13 in BC progression (Fig. 3D and E). Moreover, KIF18B knockdown significantly decreased the TRIP13 expression levels in HCC-1937 cells (Fig. 3F). The direct interaction between TRIP13 and KIF18B was determined using a Co-IP assay (Fig. 3G). These findings indicated that KIF18B may play an oncogenic role in BC by binding to TRIP13 and positively regulating TRIP13 expression levels.

Overexpression of TRIP13 attenuates the inhibitory effects of KIF18B knockdown on BC tumorigenesis. To verify the interaction between TRIP13 and KIF18B, TRIP13 overexpression 
A

A

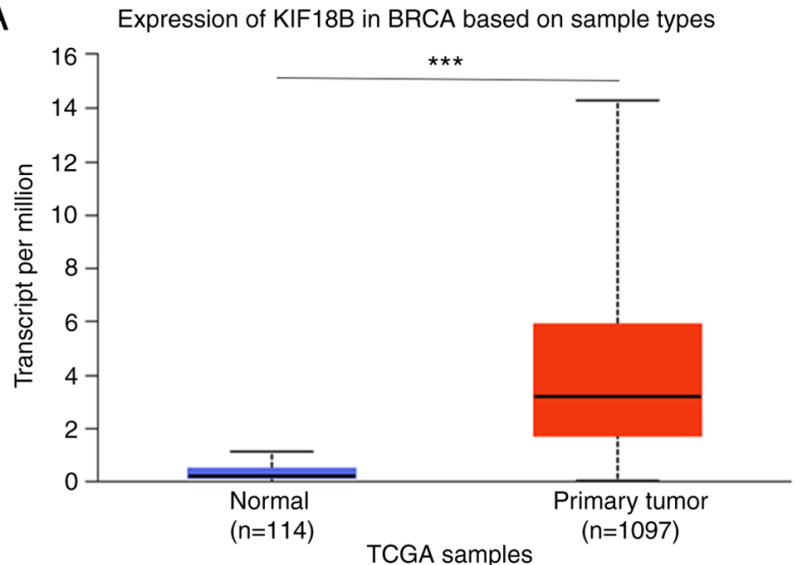

C

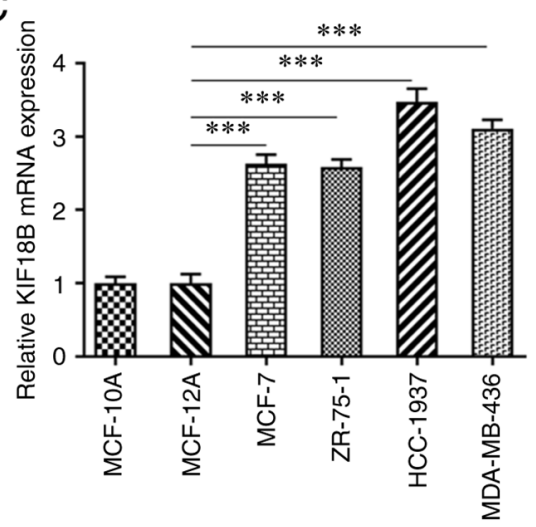

B Expression of KIF18B in BRCA based on breast cancer subclasses
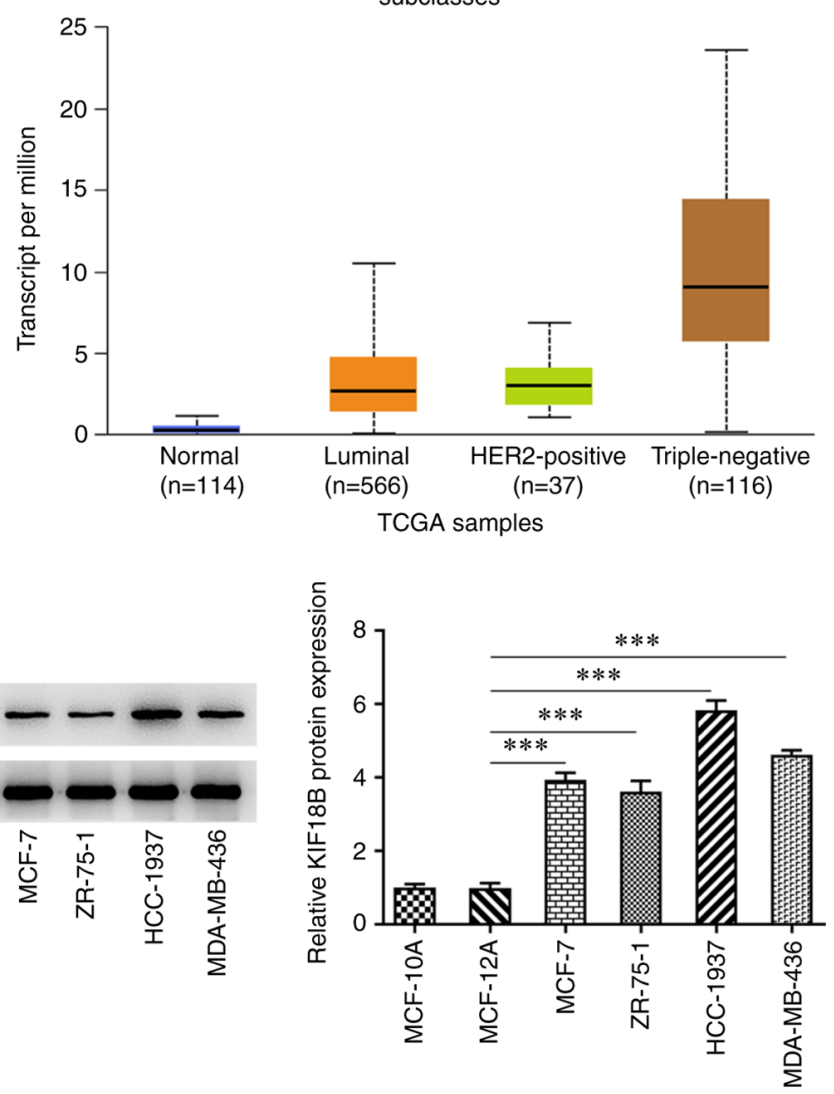

Figure 1. KIF18B overexpression in BC samples and cell lines. (A and B) KIF18 mRNA levels in BC based on sample types and subclasses were assessed by analyzing The Cancer Genome Atlas data using the UALCAN online database. (C) Reverse transcription-quantitative PCR and (D) western blot analysis were applied for the evaluation of the KIF18B mRNA and protein levels in MCF-10A and MCF-12A normal breast epithelial cell lines, and the BC cell lines, including MCF-7, ZR-75-1, HCC-1937 and MDA-MB-436. ${ }^{* * *} \mathrm{P}<0.001$. KIF18B, KIF member 18B; BC, breast cancer.

was induced in the HCC-1937 cells via transfection with Ov-TRIP13 vector (Fig. 4A and B). The control HCC-1937 cell group or the KIF18B knockdown group were co-transfected with Ov-TRIP13. Transfection with shRNA-KIF18B markedly decreased cell viability in the control cell group; however, as compared with the Ov-NC group, cells that were co-transfected with Ov-TRIP13 exhibited an increased cell viability (Fig. 4C). Similarly, the decreased number of colonies induced by KIF18B knockdown was reversed by Ov-TRIP13 (Fig. 4D). Furthermore, the impaired migratory and invasive abilities induced by KIF18B knockdown were attenuated by Ov-TRIP13 (Fig. 4E and F). KIF18B knockdown also resulted in decreased MMP12 and MMP9 protein expression levels, whereas Ov-TRIP13 reversed this effect (Fig. 4G).

Wnt/ $\beta$-catenin signaling pathway is involved in the effects of the KIF18B/TRIP13 axis on BC. $\beta$-catenin signaling pathway protein expression levels were also determined (Figure 5). The results demonstrated that KIF18B knockdown resulted in the decreased expression of $\beta$-catenin, c-Myc and cyclin D1, in comparison with the negative control. However, compared with the shRNA-KIF18B + Ov-NC cell group, the shRNA-KIF18B + Ov-TRIP13 cell group exhibited significantly higher $\mathrm{Wnt} / \beta$-catenin signaling pathway protein expression levels. These results therefore indicated that KIF18B knockdown induced the inactivation of the $\mathrm{Wnt} / \beta$-catenin signaling pathway, whereas TRIP13 overexpression re-activated this signaling pathway in HCC-1937 BC cells.

\section{Discussion}

$\mathrm{BC}$ is a common type of cancer that affects women globally. In the present study, the role of the KIF18B/TRIP13 axis in BC was explored. The results demonstrated that the expression levels of KIF18B and TRIP13 were increased in BC. It was demonstrated that KIF18B promoted BC cell malignant progression by targeting TRIP13, positively regulating TRIP13 expression levels, thereby activating the Wnt/ $\beta$-catenin signaling pathway. Therefore, the present study indicated a novel mechanism, to the best of our knowledge, involving KIF18B into driving BC progression.

KIF18B has been reported to be upregulated in BC samples and has been associated with reduced survival rates (11). In line with the previously published results, it was demonstrated in the present study that KIF18B expression was upregulated in BC cell lines. Furthermore, it was determined in silico that KIF18B expression was particularly upregulated in patients with the TNBC subtype, and this finding was further validated by in vitro experiments, with $\mathrm{KIF} 18 \mathrm{~B}$ displaying the highest expression levels in the TNBC HCC-1937 cell line. BC is a heterogeneous disease and can be categorized into three groups: i) Hormone receptor-expressing [estrogen receptor 
A

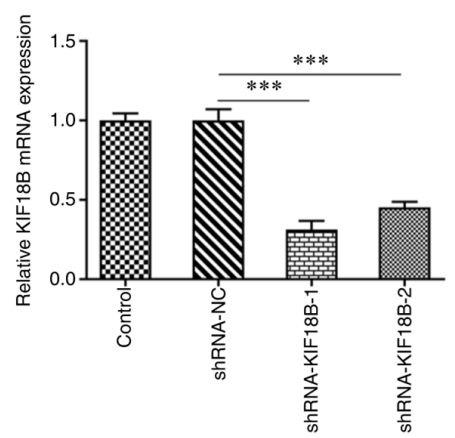

C

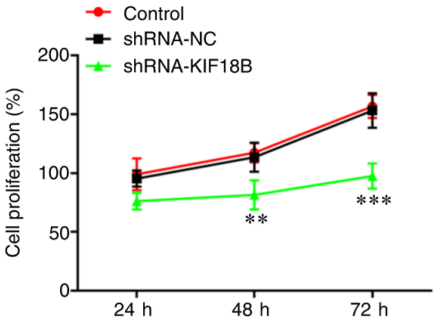

E

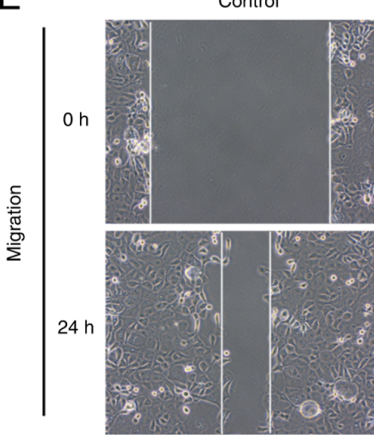

F

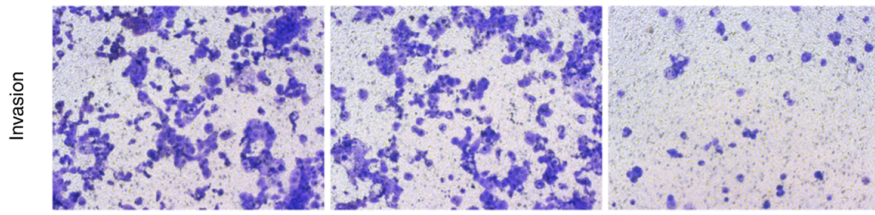

G

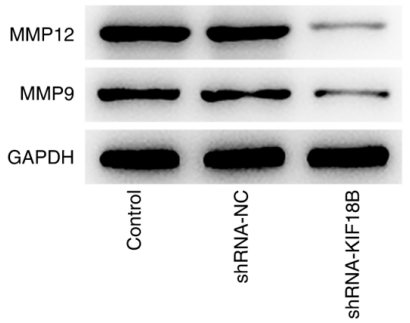

B

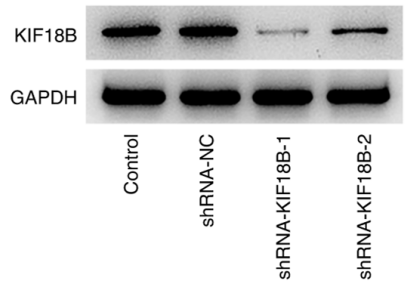

D
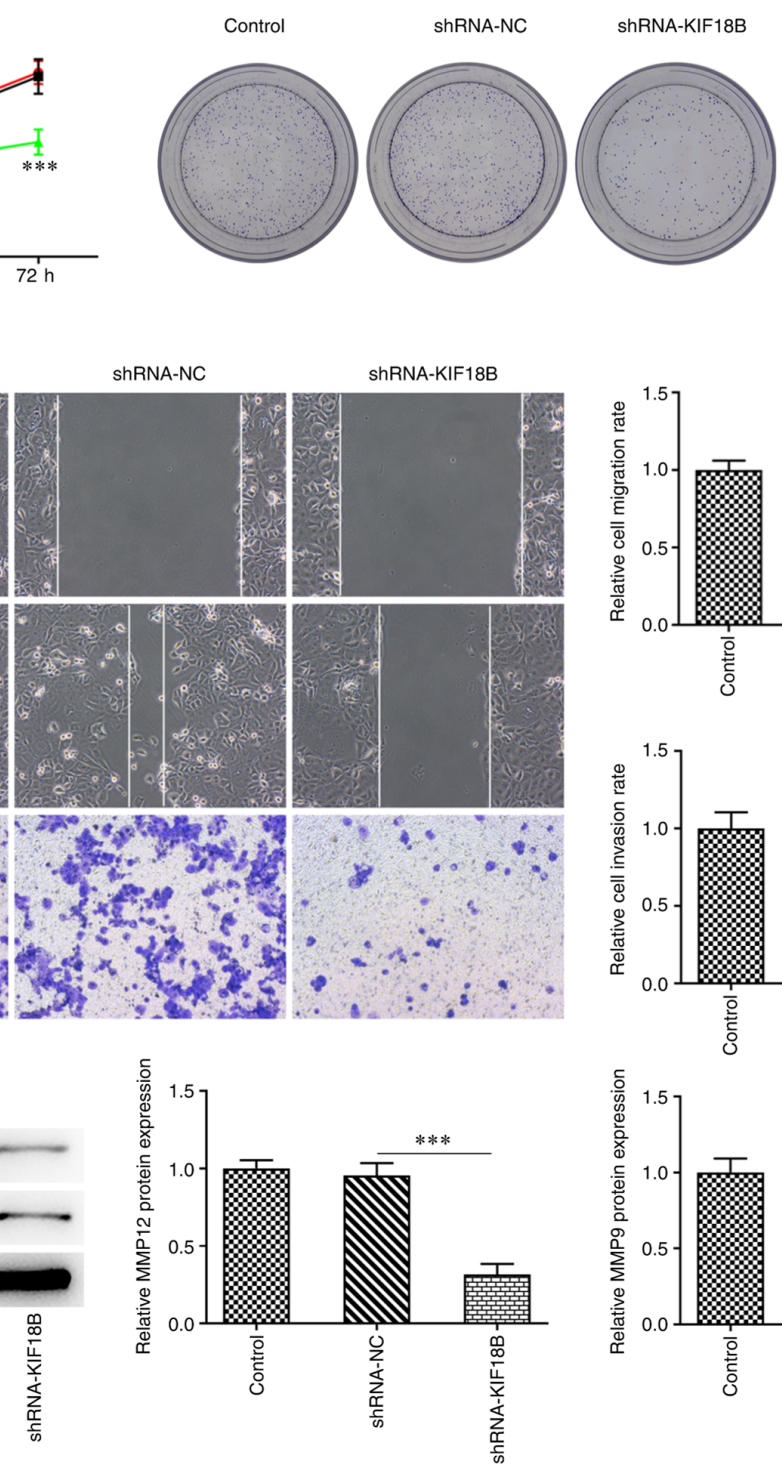
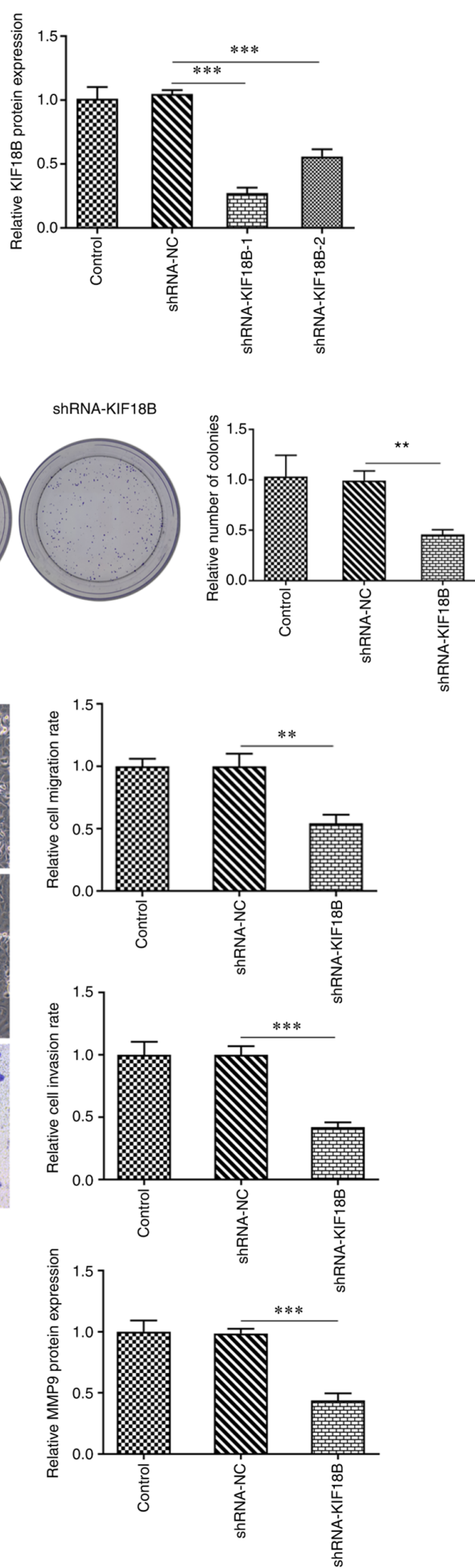

Figure 2. KIF18B knockdown inhibits the malignant progression of HCC-1937 cells. (A) KIF18B mRNA levels in transfected HCC-1937 cells, measured using reverse transcription-quantitative PCR. (B) KIF18B protein levels in transfected HCC-1937 cells, measured using western blot analysis. (C) Transfected HCC-1937 cell viability at 24, 48 and $72 \mathrm{~h}$ post-transfection, measured using CCK-8 assay. (D) Transfected HCC-1937 cell proliferation at 48 h post-transfection, measured using colony formation assay (magnification, x200). (E) Transfected HCC-1937 cell migration evaluation using wound healing assay (magnification, x200). (F) Transfected HCC-1937 cell invasion evaluation using Transwell assay (magnification, x200); (G) MMP12 and MMP9 protein expression was detected by western blotting assay. ${ }^{* * *} \mathrm{P}<0.01$ and ${ }^{* * * * *} \mathrm{P}<0.001$. KIF18B, KIF member $18 \mathrm{~B}$.

$\left(\mathrm{ER}^{+}\right)$or progesterone receptor $\left.\left(\mathrm{PR}^{+}\right)\right]$; ii) HER2-expressing $\left(\mathrm{HER}^{+}\right)$; and TNBC (ER', $\mathrm{PR}^{-}$and HER2-), which is particularly aggressive and is associated with a poor prognosis and high heterogeneity $(20,21)$. The role of KIF18B in HCC-1937 cell aggression was therefore investigated. Subsequent functional analysis revealed that KIF18B knockdown effectively inhibited HCC-1937 cell proliferation, migration and invasion. The invasion of $\mathrm{BC}$ cells into the surrounding extracellular matrix and their subsequent migration into the bloodstream and lymphatic system are pivotal steps in BC 

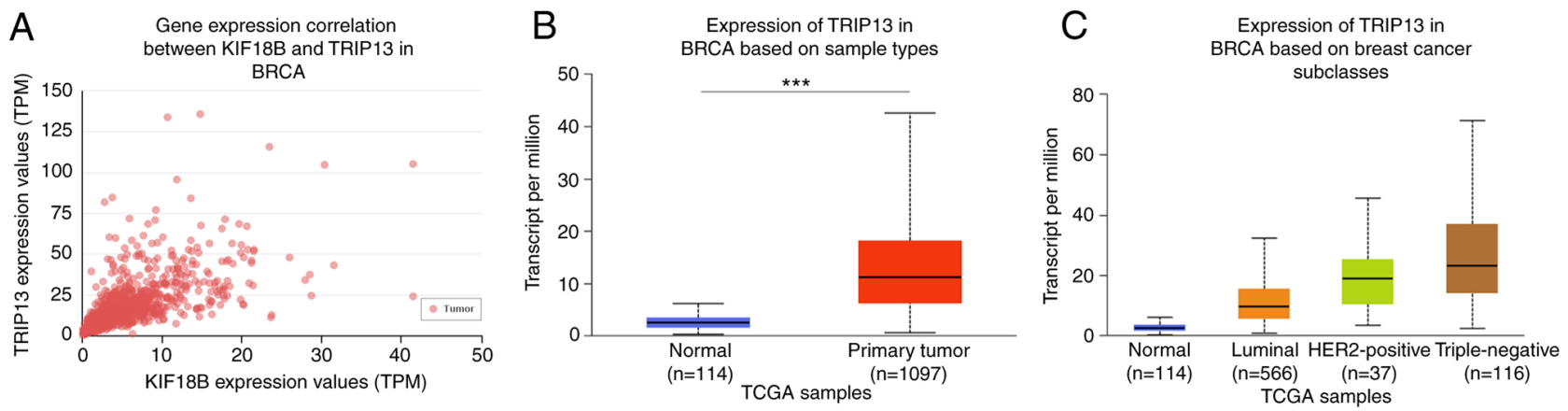
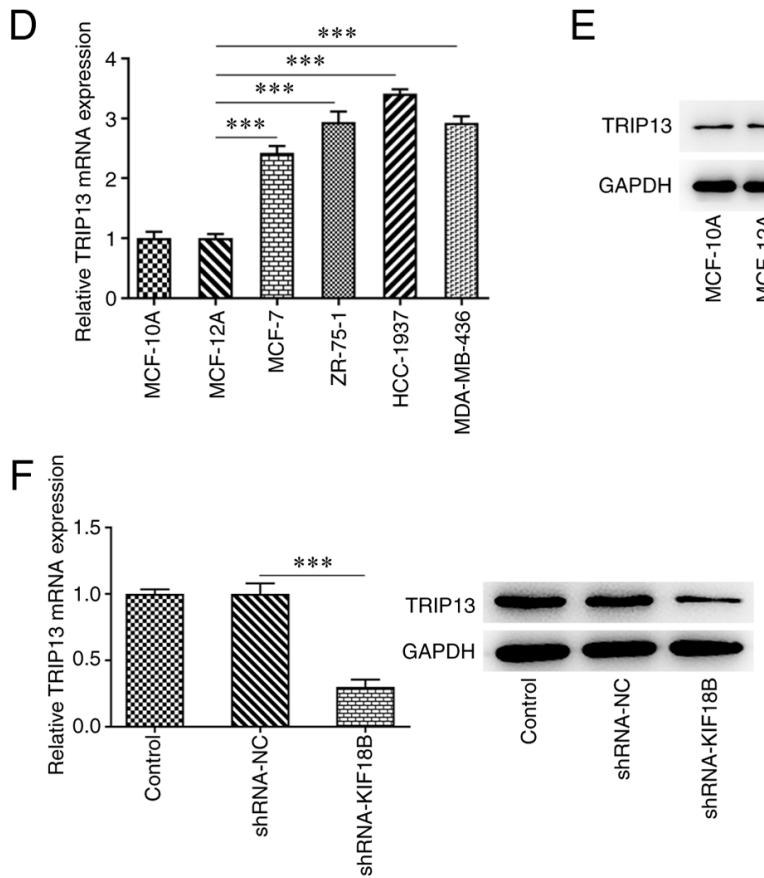

E

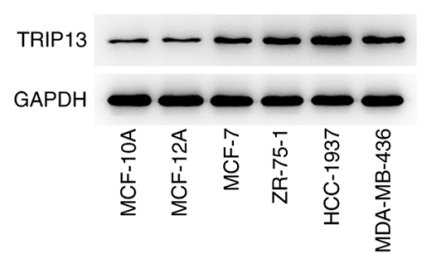

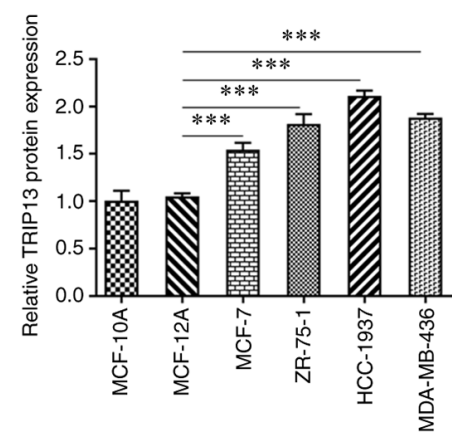

G

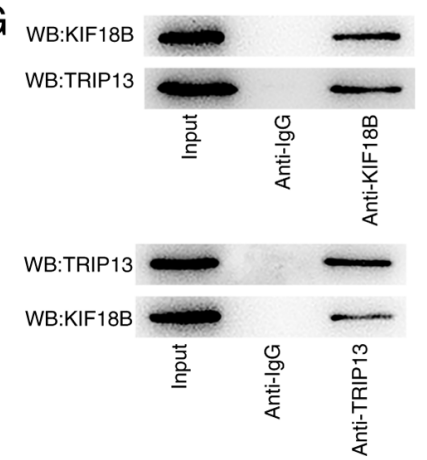

Figure 3. TRIP13 is overexpressed in BC and is positively regulated by KIF18B via direct binding. (A) Correlation between KIF18B and TRIP13 expression in BC. (B) TRIP13 mRNA level evaluation in BC, based on sample types, by analyzing TCGA data using online databases UALCAN and subclasses. (C) TRIP13 mRNA level evaluation in BC, based on subclasses, by analyzing TCGA data using the UALCAN online database. (D) TRIP13 mRNA expression evaluation and (E) TRIP13 protein expression evaluation using reverse transcription-quantitative PCR and western blot analysis, respectively, in MCF-10A and MCF-12A normal breast epithelial cell lines, and the BC cell lines, including MCF-7, ZR-75-1, HCC-1937 and MDA-MB-436. (F) TRIP13 mRNA and protein expression evaluation in HCC-1937 cells with or without KIF18B. (G) Co-IP assay was used to determine the protein interaction between KIF18B and TRIP13. ${ }^{* * *} \mathrm{P}<0.001$. KIF18B, KIF member 18B; TRIP13, thyroid hormone receptor-interacting protein 13; BC, breast cancer; Co-IP, co-immunoprecipitation. TCGA, The Cancer Genome Atlas.

metastasis (22). The results of the present study suggested the potential value of KIF18B knockdown in the treatment of BC, particularly TNBC.

To explore the underlying mechanisms of KIF18B in mediating $\mathrm{BC}$ tumorigenesis, the factors closely associated with KIF18B expression in BC were explored. TRIP13 was revealed to be positively associated with KIF18B expression in $\mathrm{BC}$ and exhibited a similar expression profile to that of $\mathrm{KIF} 18 \mathrm{~B}$, in which it was upregulated in BC, particularly in the TNBC subtype. Similar to KIF18B, TRIP13 was also most highly expressed in the HCC-1937 cell line. Moreover, the results of the present study validated that KIF18B was able to bind to TRIP13 and positively regulate its expression levels. These results strongly suggested the involvement of TRIP13 in KIF18B-mediated BC tumorigenesis. Further results revealed that TRIP13 overexpression markedly abolished the inhibitory effect of KIF18B knockdown on HCC-1937 cell proliferation, migration and invasion, further suggesting that TRIP13 had an indispensable role in KIF18B-mediated BC tumorigenesis.

KIF18B has previously been reported to activate the Wnt/ $\beta$-catenin signaling pathway, promoting the tumor progression of hepatocellular carcinoma (23), cervical cancer (24) and BC (11). In the present study, it was demonstrated that the decreased $\beta$-catenin, c-Myc and cyclin D1 protein expression levels in HCC-1937 cells were attributed to KIF18B knockdown. The Wnt/ $\beta$-catenin signaling pathway has been reported to play a crucial role in cancer cell proliferation, survival, migration and invasion (25). When Wnt ligands bind to transmembrane receptors, Wnt signaling is initiated. Cyclin D1 is the upstream signaling molecule of $\beta$-catenin and c-Myc is one of the target genes of $\beta$-catenin (26). The results of the present study demonstrated that KIF18B functioned as an oncogene in $\mathrm{BC}$ via the activation of the Wnt/ $\beta$-catenin signaling pathway, and therefore promoted the 
A

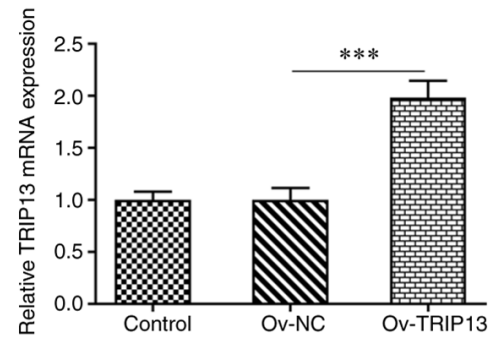

B

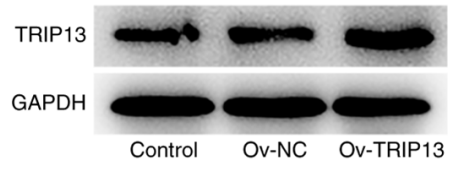

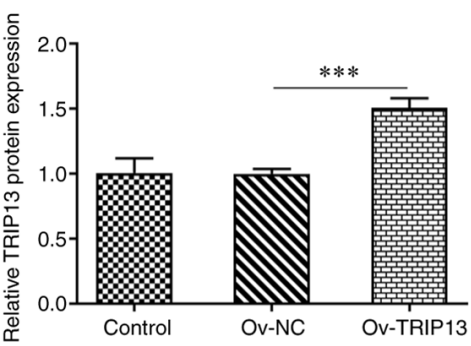

C

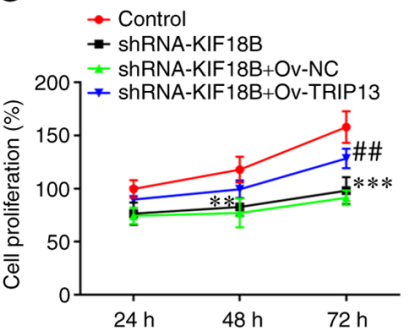

D
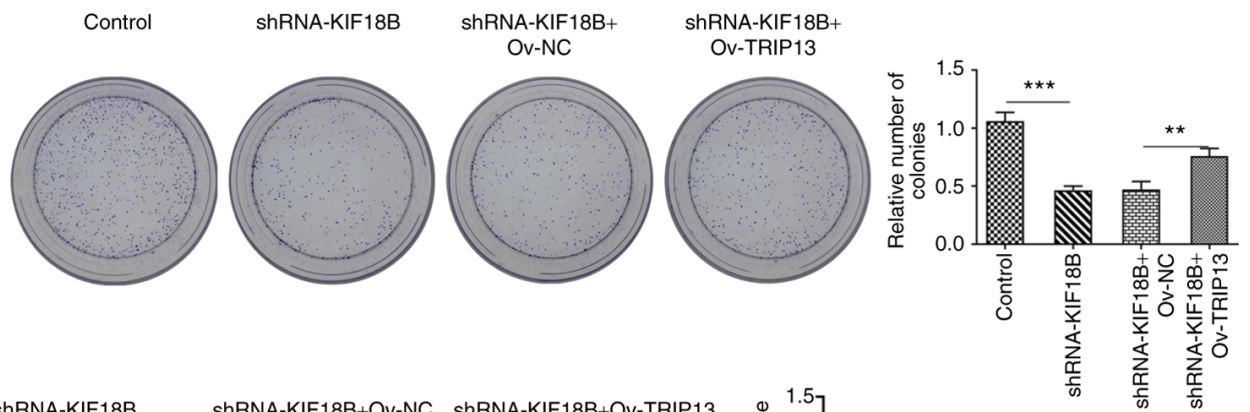

E
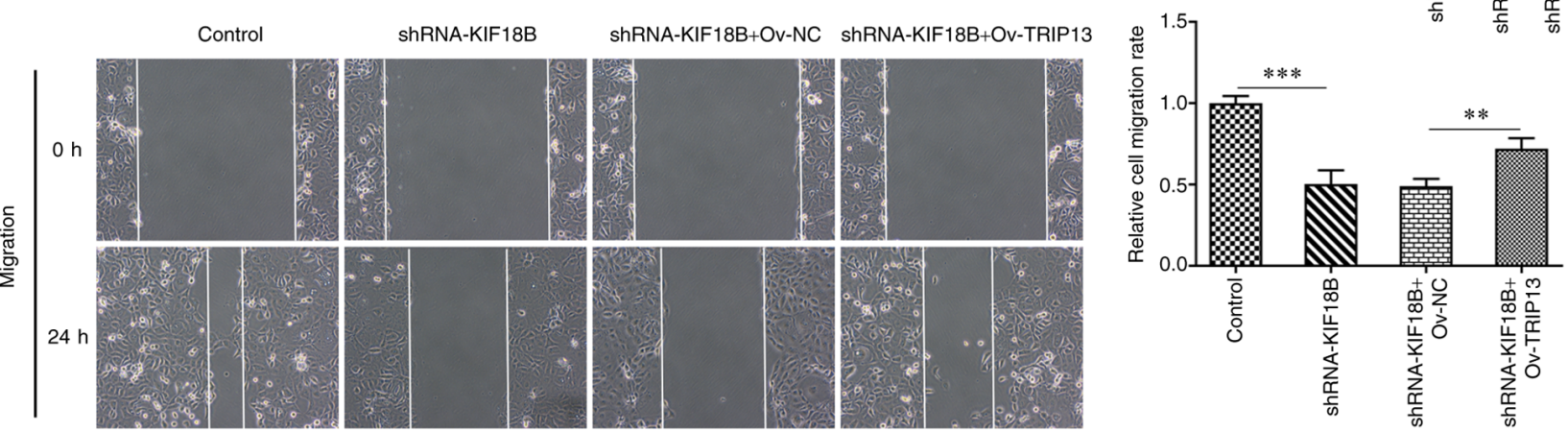

$\mathrm{F}$
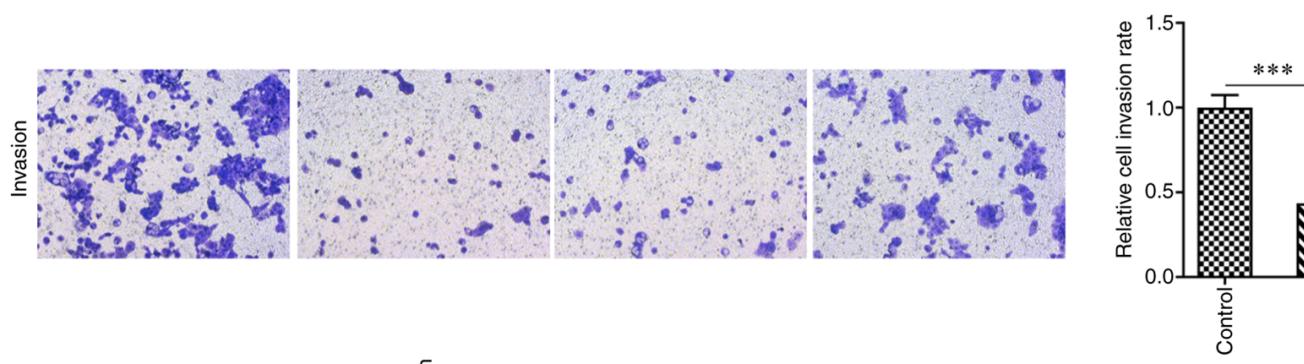

G
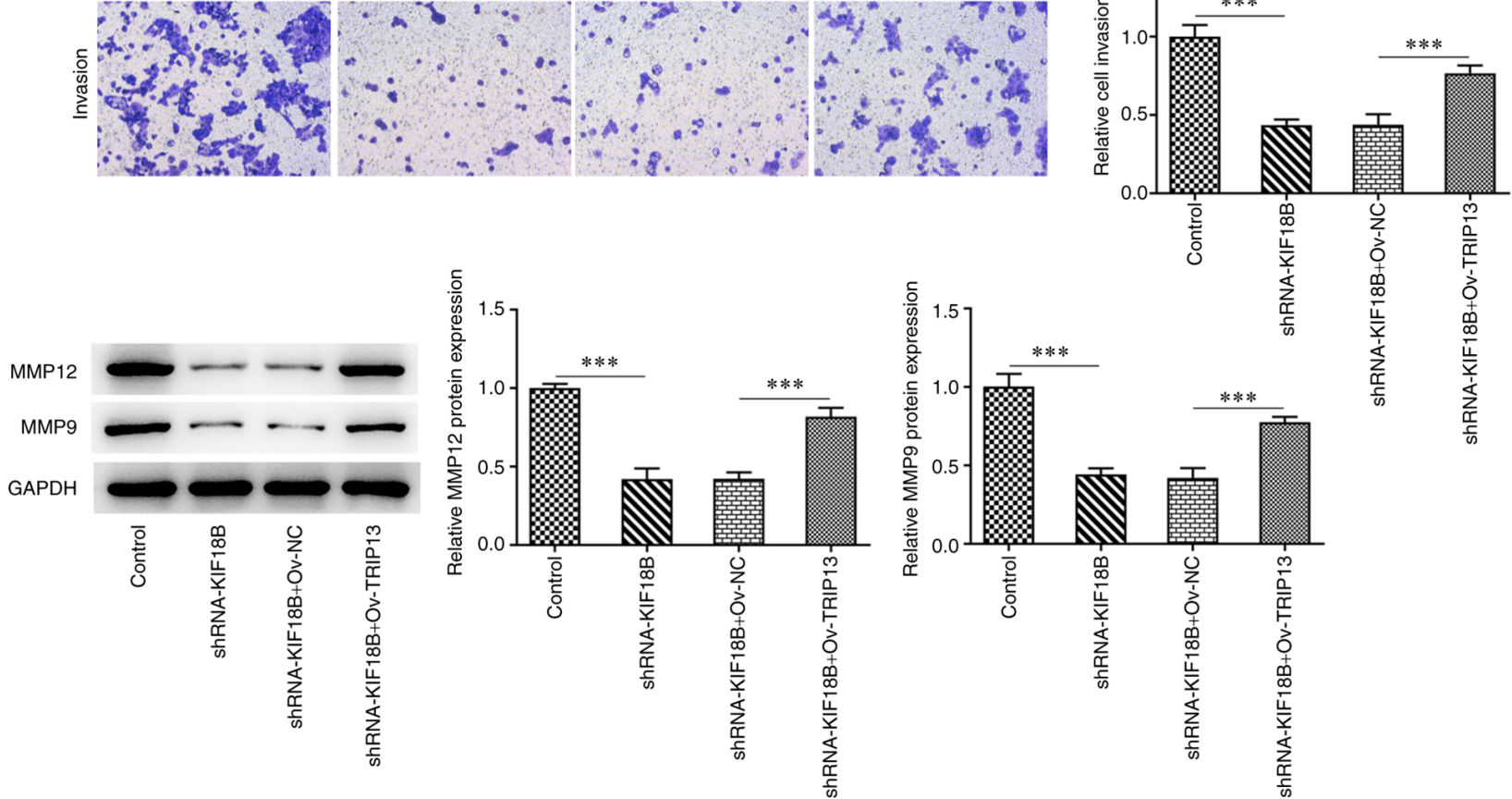

Figure 4. TRIP13 overexpression attenuates the inhibitory effects of KIF18B knockdown on HCC-1937 cells progression. (A) TRIP13 mRNA level evaluation using reverse transcription-quantitative PCR in transfected HCC-1937 cells. (B) TRIP13 protein level evaluation using western blot analysis, in transfected HCC-1937 cells. ${ }^{* * *} \mathrm{P}<0.001$. (C) Cell viability evaluation in transfected HCC-1937 cells at 24,48 and $72 \mathrm{~h}$ post-transfection, using CCK-8 assay. ${ }^{* *} \mathrm{P}<0.01$ and ${ }^{* * * *} \mathrm{P}<0.001$, vs. control; ${ }^{\# \#} \mathrm{P}<0.01$, vs. shRNA-KIF18B + Ov-NC. (D) Cell proliferation evaluation using colony formation assay. (E) Cell migration evaluation, using wound healing assay. (F) Cell invasion evaluation, using Transwell assay. (G) MMP12 and MMP9 protein expression evaluation using western blot analysis. ${ }^{* *} \mathrm{P}<0.01$ and ${ }^{* * *} \mathrm{P}<0.001$. KIF18B, KIF member 18B; TRIP13, thyroid hormone receptor-interacting protein 13; ov-NC, overexpression negative control. 

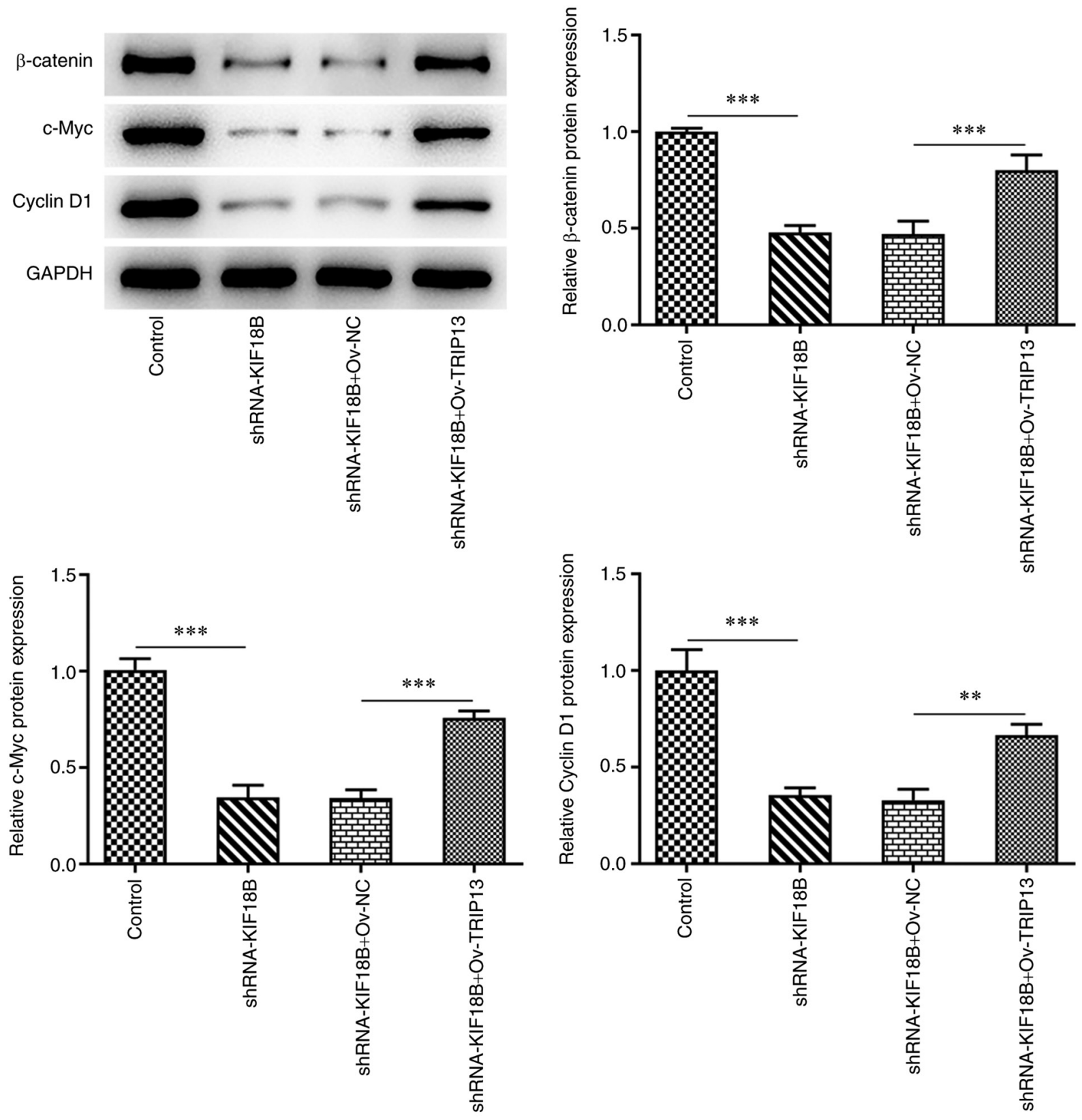

Figure 5. KIF18B knockdown-mediated inactivation of Wnt/ $\beta$-catenin signaling is reversed by TRIP13 overexpression. Transfected and untransfected HCC-1937 cells were used, and the $\beta$-catenin, c-Myc and cyclin D1 protein expression was examined using western blot analysis. ${ }^{* *} \mathrm{P}<0.01$ and ${ }^{* * *} \mathrm{P}<0.001$. KIF18B, KIF member 18B; TRIP13, thyroid hormone receptor-interacting protein 13.

migration and invasion of HCC-1937 cells. Furthermore, it was demonstrated that TRIP13 overexpression reactivated the Wnt/ $\beta$-catenin signaling pathway, previously being inactivated due to KIF18B knockdown. The TRIP13-mediated activation of the Wnt/ $\beta$-catenin signaling pathway has also been reported in lung, colorectal and cervical cancer (27-29). It was therefore suggested that the activation of the Wnt/ $\beta$-catenin signaling pathway in BC might be also caused by TRIP13. However, further research is required, in order to validate this finding. However, this study also had several limitations. Firstly, whether KIF18B overexpression can alter the effects mediated by TRIP13 silencing on BC cell progression, as well as the underlying mechanisms, may also be worthy of investigation. Moreover, the use of other TNBC or BC cell lines and the application of in vivo experiments, using animal models alongside the evaluation of clinical patient samples are required to further validate the conclusions of the present study. In addition, although the inhibition on cancer cell proliferation, migration and invasion is sufficient to illustrate the anti-cancer effect in vitro $(30,31)$, the involvement of cell death and apoptosis has to be also assessed in vitro and in vivo, to further confirm this effect in future research.

In conclusion, the present study indicated that KIF18B/TRIP13 was upregulated in BC, particularly in the TNBC subtype, in both cell lines and tissues. Furthermore, KIF18B knockdown suppressed the malignant process in TNBC cells by decreasing the TRIP13 expression levels, which may have inactivated the Wnt/ $\beta$-catenin signaling pathway. Overall, these findings suggest that KIF18B/TRIP13 may play a crucial role in $\mathrm{BC}$ tumorigenesis. Thus, this axis 
may prove to be a novel prognostic biomarker for patients with $\mathrm{BC}$ in the future.

\section{Acknowledgements}

Not applicable.

\section{Funding}

The present study was funded by the Construction of Women's Life Cycle Health Management Service Model in Baoji Area, Key R\&D Projects in Shaanxi Province (grant no. 2021SF-211).

\section{Availability of data and materials}

The datasets used and/or analyzed during the current study are available from the corresponding author on reasonable request.

\section{Authors' contributions}

$\mathrm{JL}$ and LL contributed to the conception and design of the study. LL, ZZ and XX contributed to the acquisition of data. $\mathrm{LL}$ and $\mathrm{ZZ}$ contributed to the analysis or interpretation of the data. JL and LL drafted the manuscript and revised it critically for important intellectual content. All authors have read and approved the final manuscript. JL and LL confirm the authenticity of all the raw data.

\section{Ethics approval and consent to participate}

Not applicable.

\section{Patient consent for publication}

Not applicable.

\section{Competing interests}

The authors declare that they have no competing interests.

\section{References}

1. Coughlin SS: Epidemiology of breast cancer in women. Adv Exp Med Biol 1152: 9-29, 2019.

2. Cheung KL: Treatment strategies and survival outcomes in breast cancer. Cancers (Basel) 12: 735, 2020.

3. Falzone L, Grimaldi M, Celentano E, Augustin LSA and Libra M: Identification of modulated MicroRNAs associated with breast cancer, diet, and physical activity. Cancers (Basel) 12: 2555, 2020.

4. Arnedos M, Roulleaux Dugage M, Perez-Garcia J and Cortes J: Window of opportunity trials for biomarker discovery in breast cancer. Curr Opin Oncol 31: 486-492, 2019.

5. Emens LA: Breast cancer immunotherapy: Facts and hopes. Clin Cancer Res 24: 511-520, 2018.

6. Zhong A, Tan FQ and Yang WX: Chromokinesin: Kinesin superfamily regulating cell division through chromosome and spindle. Gene 589: 43-48, 2016.

7. Hirokawa $\mathrm{N}$ and Tanaka Y: Kinesin superfamily proteins (KIFs): Various functions and their relevance for important phenomena in life and diseases. Exp Cell Res 334: 16-25, 2015.

8. Lucanus AJ and Yip GW: Kinesin superfamily: Roles in breast cancer, patient prognosis and therapeutics. Oncogene 37: 833-838, 2018.

9. Fu Y, Zhou QZ, Zhang XL, Wang ZZ and Wang P: Identification of Hub genes using co-expression network analysis in breast cancer as a tool to predict different stages. Med Sci Monit 25: 8873-8890, 2019.
10. Li TF, Zeng HJ, Shan Z, Ye RY, Cheang TY, Zhang YJ, Lu SH, Zhang Q, Shao N and Lin Y: Overexpression of kinesin superfamily members as prognostic biomarkers of breast cancer. Cancer Cell Int 20: 123, 2020.

11. Jiang J, Liu T, He X, Ma W, Wang J, Zhou Q, Li M and Yu S: Silencing of KIF18B restricts proliferation and invasion and enhances the chemosensitivity of breast cancer via modulating

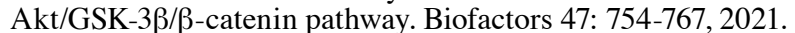

12. Miniowitz-Shemtov S, Eytan E, Kaisari S, Sitry-Shevah D and Hershko A: Mode of interaction of TRIP13 AAA-ATPase with the Mad2-binding protein p31comet and with mitotic checkpoint complexes. Proc Natl Acad Sci USA 112: 11536-11540, 2015.

13. Lu S, Qian J, Guo M, Gu C and Yang Y: Insights into a Crucial Role of TRIP13 in human cancer. Comput Struct Biotechnol J 17: 854-861, 2019.

14. Dazhi W, Mengxi Z, Fufeng C and Meixing Y: Elevated expression of thyroid hormone receptor-interacting protein 13 drives tumorigenesis and affects clinical outcome. Biomark Med 11: 19-31, 2017.

15. Wang K, Sturt-Gillespie B, Hittle JC, Macdonald D, Chan GK, Yen TJ and Liu ST: Thyroid hormone receptor interacting protein 13 (TRIP13) AAA-ATPase is a novel mitotic checkpoint-silencing protein. J Biol Chem 289: 23928-23937, 2014.

16. Vedoya GM, López Nigro MM and Martín GA: The secretome of non-tumorigenic mammary cells MCF-10A elicits DNA damage in MCF-7 and MDA-MB-231 breast cancer cells. Toxicol In Vitro 70: 105018, 2021.

17. van den Brand AD, Villevoye J, Nijmeijer SM, van den Berg M and van Duursen MBM: Anti-tumor properties of methoxylated analogues of resveratrol in malignant MCF-7 but not in non-tumorigenic MCF-10A mammary epithelial cell lines. Toxicology 422: 35-43, 2019.

18. Livak KJ and Schmittgen TD: Analysis of relative gene expression data using real-time quantitative PCR and the 2(-Delta Delta C(T)) Method. Methods 25: 402-408, 2001.

19. Gobin E, Bagwell K, Wagner J, Mysona D, Sandirasegarane S, Smith N, Bai S, Sharma A, Schleifer R and She JX: A pan-cancer perspective of matrix metalloproteases (MMP) gene expression profile and their diagnostic/prognostic potential. BMC Cancer 19: 581, 2019.

20. Barzaman K, Karami J, Zarei Z, Hosseinzadeh A, Kazemi MH, Moradi-Kalbolandi S, Safari E and Farahmand L: Breast cancer: Biology, biomarkers, and treatments. Int Immunopharmacol 84: 106535, 2020.

21. Tsang JYS and Tse GM: Molecular classification of breast cancer. Adv Anat Pathol 27: 27-35, 2020.

22. Lyons TG: Targeted therapies for triple-negative breast cancer. Curr Treat Options Oncol 20: 82, 2019.

23. Yang B, Wang S, Xie H, Wang C, Gao X, Rong Y, Liu Z and Lu Y: KIF18B promotes hepatocellular carcinoma progression through activating Wnt/ $\beta$-catenin-signaling pathway. J Cell Physiol 235: 6507-6514, 2020.

24. Wu Y, Wang A, Zhu B, Huang J, Lu E, Xu H, Xia W, Dong G, Jiang $\mathrm{F}$ and Xu L: KIF18B promotes tumor progression through activating the Wnt/ $\beta$-catenin pathway in cervical cancer. Onco Targets Ther 11: 1707-1720, 2018.

25. Krishnamurthy $\mathrm{N}$ and Kurzrock R: Targeting the Wnt/betacatenin pathway in cancer: Update on effectors and inhibitors. Cancer Treat Rev 62: 50-60, 2018.

26. Tian D, Tian M, Ma ZM,Zhang LL, Cui YF and Li JL: Anesthetic propofol epigenetically regulates breast cancer trastuzumab resistance through IL-6/miR-149-5p axis. Sci Rep 10: 8858, 2020.

27. Li ZH, Lei L, Fei LR, Huang WJ, Zheng YW, Yang MQ, Wang Z, Liu CC and Xu HT: TRIP13 promotes the proliferation and invasion of lung cancer cells via the Wnt signaling pathway and epithelial-mesenchymal transition. J Mol Histol 52: 11-20, 2021.

28. Agarwal S, Behring M, Kim HG, Chandrashekar DS, Chakravarthi BVSK, Gupta N, Bajpai P, Elkholy A, Al Diffalha S, Datta PK, et al: TRIP13 promotes metastasis of colorectal cancer regardless of p53 and microsatellite instability status. Mol Oncol 14: 3007-3029, 2020.

29. Liu X, Shen X and Zhang J: TRIP13 exerts a cancer-promoting role in cervical cancer by enhancing Wnt/ $\beta$-catenin signaling via ACTN4. Environ Toxicol 36: 1829-1840, 2021.

30. Zhu J, Zhou L, Wei B, Qian Z, Wang J, Hui H and Sun Y: MiR-142-5p inhibits pancreatic cancer cell migration and invasion by targeting PIK3CA. Mol Med Rep 22: 2085-2092, 2020.

31. Yang S, Sun S, Xu W, Yu B, Wang G and Wang H: Astragalus polysaccharide inhibits breast cancer cell migration and invasion by regulating epithelial-mesenchymal transition via the $\mathrm{Wnt} / \beta$-catenin signaling pathway. Mol Med Rep 21: 1819-1832, 2020.

This work is licensed under a Creative Commons Attribution-NonCommercial-NoDerivatives 4.0 International (CC BY-NC-ND 4.0) License. 